\title{
Determining Actuator Requirements for Cyclic Varying Pitch Propeller for Ships
}

\author{
Uffe Sjølund Freiberg ${ }^{1}$, Torben O. Andersen ${ }^{1}$, Jens Ring Nielsen ${ }^{2}$ and Henrik C. Pedersen ${ }^{1, *}$ (D) \\ 1 AAU Energy, Aalborg University, 9000 Aalborg, Denmark; usf@et.aau.dk (U.S.F.); toa@energy.aau.dk (T.O.A.) \\ 2 MAN Energy Solutions, 9900 Frederikshavn, Denmark; jensr.nielsen@es-man.com \\ * Correspondence: hcp@energy.aau.dk
}

Citation: Freiberg, U.S.; Andersen, T.O.; Nielsen, J.R.; Pedersen, H.C. Determining Actuator Requirements for Cyclic Varying Pitch Propeller for Ships. Energies 2021, 14, 6554. https://doi.org/10.3390/en14206554

Academic Editor: Kyung Chun Kim

Received: 1 July 2021

Accepted: 22 September 2021

Published: 12 October 2021

Publisher's Note: MDPI stays neutral with regard to jurisdictional claims in published maps and institutional affiliations.

Copyright: () 2021 by the authors. Licensee MDPI, Basel, Switzerland. This article is an open access article distributed under the terms and conditions of the Creative Commons Attribution (CC BY) license (https:// creativecommons.org/licenses/by/ $4.0 /)$.

\begin{abstract}
In marine applications, a cyclic varying pitch (CVP) propeller is a propeller in which the propeller blade can be cyclic-pitched. This cyclic pitching of the propeller blades is used to adapt to the local flow conditions in the non-uniform wake field that the propeller operates in, behind the ship hull. This has the potential to improve the performance of the propulsion system relative to a propeller which has fixed pitch for each revolution. The potential performance improvements include increasing the propulsion efficiency and reducing the cavitation, pressure pulses, vibrations and noise problems. However, the CVP propeller is not on the market today, and several challenges have to be addressed before the CVP propeller may be realized. One of these challenges is how to design the individual cyclic pitch mechanism for the propeller. However, before the cyclic pitch mechanism can be designed, it is necessary to know the requirements for it, such as the required pitching power and torque. The focus of the current paper is therefore to present a model for the propeller, by which it is possible to determine the loads acting on the CVP propeller blades during the cyclic pitching, and hence the actuator force/torque and power requirements. To illustrate the usefulness of the model, an example is presented, in which the loads on a CVP propeller are determined, together with the requirements for the individual cyclic pitch mechanism. The efficiency results presented are, however, not representative of the efficiency improvement that may be obtained, as neither the propeller nor the pitch trajectory has been optimised. The results do, however, serve to show the benefit and validity of the model.
\end{abstract}

Keywords: cyclic varying pitch; propeller; ship

\section{Introduction}

A cyclic varying pitch (CVP) propeller is a propeller for ship propulsion that can pitch the propeller blades cyclically, i.e., the propeller pitch may follow a given trajectory for a single revolution. On the contrary, conventional propellers currently used for ship propulsion are fixed-pitch (FP) propellers or controllable pitch (CP) propellers. Both these propellers have a fixed pitch for a given revolution, but the pitch angle may change simultaneously for all blades for the CP propeller. Thus, the propeller placement behind the ship hull means that the flow through the propeller is non-uniformly distributed. This non-uniform flow field is also called the wake field of the ship, and an example is seen in Figure 1 for a single screw propeller. The wake field in the figure is normalized relative to the ship's speed, $V_{s}$, where $v_{a}$ is the velocity of the incoming water along the forward direction of the ship (usually termed the local advance velocity). From Figure 1, we can see that the velocity distribution varies radially and circumferentially. A minimum of the fluid velocity is around the top of the wake field, i.e., the 12 o'clock position, and the maximum is around the bottom of the wake field, i.e., the 6 o'clock position. 


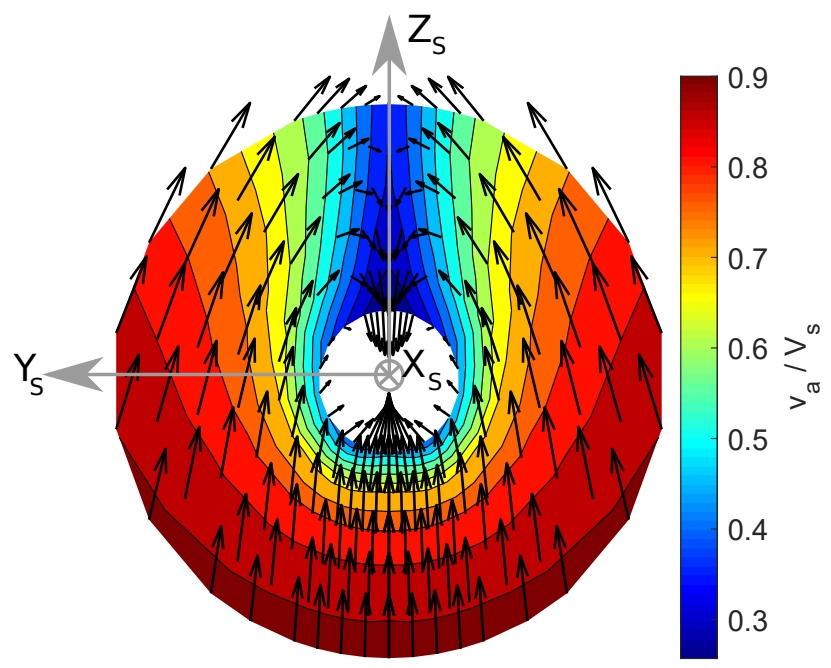

Figure 1. Wake field for a single screw ship. Reproduced from [1] with permission from the authors.

The non-uniform wake field introduces some problems for the FP and CP propellers, since the local flow conditions around the propeller blade depend on its position in the wake field. These problems relate to vibrations and transient cavitation, which must be compensated for when designing these propeller blades. However, reducing the vibrations and transient cavitation typically comes at the cost of reduced propeller efficiency.

The CVP propeller changes the blades pitch cyclic to adapt to the local flow conditions of the non-uniform wake field. The cyclic pitching with the CVP propeller is thus used to compensate for the non-uniform wake field, as illustrated in Figure 2.

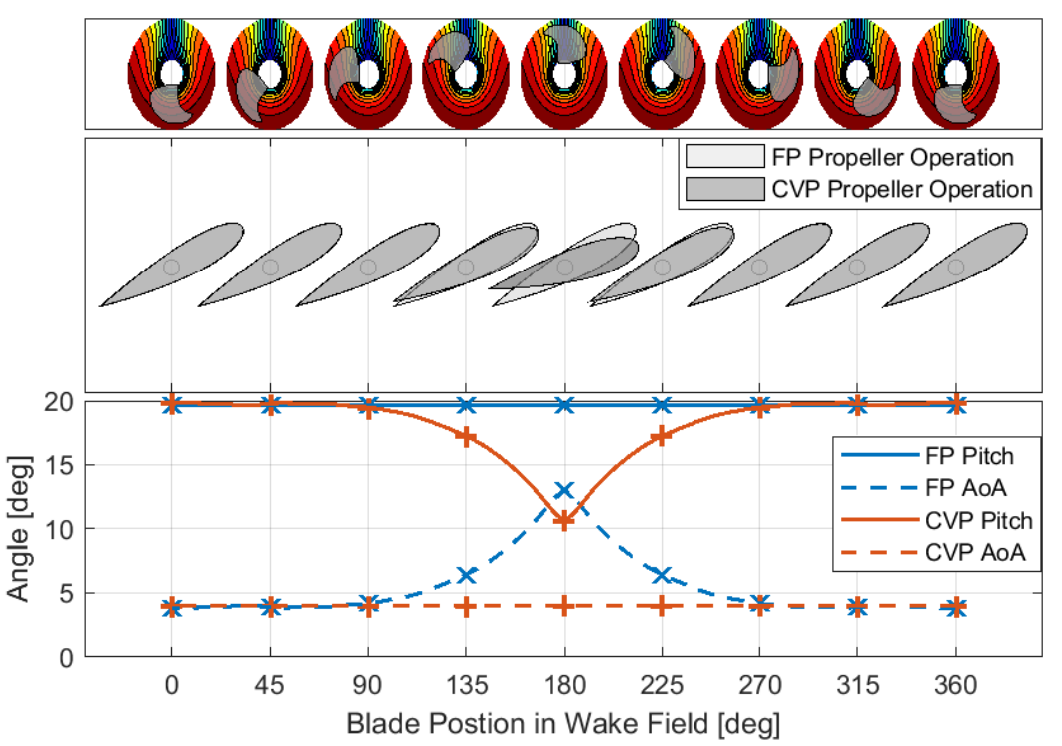

Figure 2. Illustration of the operation principle of the CVP propeller. Reproduced from [1] with permission from the authors. AoA is an abbreviation for angle of attack.

The top plot in Figure 2 shows the position of the blade in the wake field as the propeller rotates. The middle plot shows a cross-sectional view of one of the foil sections of the propeller blade for a CVP and a $\mathrm{CP}$ propeller (for the $\mathrm{CP}$ propeller, the pitch angle is fixed; this is why "fixed pitch" is denoted in the figure). From this foil section view, it is seen that the pitch of the foil section for the CVP propeller decrease around the blade top position, while the pitch for the fixed $\mathrm{CP}$ propeller is constant. The bottom plot in Figure 2 shows the pitch of the considered foil section as solid lines and the angle of attack using the dashed line. It is seen that, for the fixed CP propeller, the pitch is constant, while 
the angle of attack increases around the wake peak. For the CVP propeller, it is seen that the angle of attack is constant, while the pitch varies around the wake peak. This should lead to reduced shaft vibrations, increased efficiency and reduced transient cavitation in the wake peak. The illustration only considers one radial section of the propeller blade. Therefore, the optimum pitch trajectory for the whole blade will be a trade-off between the performance of each radial section of the propeller blade.

The potential performance improvement with the CVP propeller was reviewed in [1], together with a review of the different proposed concepts for the individual cyclic pitch mechanism. In [2-8], a reduction of the cavitation is shown with the CVP propeller, and efficiency improvements are obtained in $[2,5,7,8]$. The studies [2,7] considered using the CVP propeller instead of a CP propeller, but with the same geometry of the propeller blades. The efficiency improvements obtained in [2,7] are from 1.5-3\%. In [8], the geometry of the propeller blade was adapted for the CVP propeller depending on the cyclic pitching motion. With this, an efficiency increase from $6.4-9.4 \%$ was obtained. There is hence a significant energy saving potential by realizing a CVP propeller, thereby helping to reduce the fuel consumption and $\mathrm{CO}_{2}$ emission from the maritime industry-an industry which, in 2012, was accountable for approximately $2.2 \%$ of the global $\mathrm{CO}_{2}$ emission [9]. However, the focus of the current paper is not on the potential efficiency improvements, but on determining the requirements for the pitch mechanism actuators, as described below.

In [1], four challenges in realizing the CVP propeller are identified, which have not yet been addressed by the maritime industry and research groups. These challenges concern the propeller blade design, pitch trajectory, individual cyclic pitch mechanism and reliability. The focus of the current paper is on the challenges related to developing the individual cyclic pitch mechanism, where the challenge is how to design a mechanism for the CVP propeller which can pitch the propeller blades individually in a cyclic manner [1]. However, to disclose this, it is first necessary to determine the requirements for the mechanism, such as the required power and torque to pitch the propeller blade according to the desired pitch motion. The focus of the current paper is hence on determining the requirements for the pitch mechanism and actuator.

One goal is to reduce the power consumption for propulsion. To obtain this, it is a requirement that the power required to pitch the propeller blades is smaller than the power savings obtained by improving the propeller efficiency. Otherwise, the benefit of the CVP propeller will only be as a cavitation reducing propeller. In that case, the potential market for the CVP propeller will change to focus on the navy vessels, cruise ships and research vessels [1], whereas otherwise, it is all ship types. Furthermore, determining the necessary power and torque is a prerequisite for making a feasibility study for the individual pitch mechanism for the CVP propeller. For the CVP propeller, such feasibility studies have been presented in $[10,11]$, but the prerequisite which $[10,11]$ is based upon is inadequate. In [10], the friction in the bearing is, for example, neglected, and in [11], the hydrodynamic modelling is inadequate. Therefore, it is necessary to make a model that may determine the required power and torque to cyclically pitch the CVP propeller blades if the CVP propeller is to be realized.

As mentioned above, this paper focuses on presenting a method to determine the required power and torque to pitch the CVP propeller blade in the desired pitch motion for a known propeller geometry and operating conditions. Compared to previous models, the main contributions are that the presented model is complete, accounting for all forces acting on the propeller, including frictional forces and all inertial effects, while incorporating a complete hydrodynamic model. Thus, it was previously neglected that we have a significant influence on the propeller and that the requirements for the pitching mechanism are included. Furthermore, the model benefits from being based on inverse rigid body motion, thus enabling the model to be solved iteratively. The method consists of identifying all the load forces and torques acting on the CVP propeller blades through a free body diagram and modelling these loads, as described in Section 2. These loads relate to the inertial loads, hydrodynamic loads, pitch mechanism loads, frictional loads, gravitational 
loads, hub loads and bearing loads. The foundation for the model is knowing the blade geometry and the operation conditions of the propeller, such as the wake field, ship speed, propeller speed, pitch trajectory, etc. The model usage is shown in Section 3, where the model is applied to an example propeller. This is the same propeller as considered in $[8,11,12]$, as no data exist for an optimized CVP propeller. Similarly, no optimized pitch trajectory current exists in the literature either. Therefore, the efficiency results presented for the example propeller analyzed are not expected to represent an optimized CVP propeller's actual or maximum obtainable propulsion efficiency. However, the focus of the current paper is not on the obtainable efficiency, but on determining the requirements for the pitching mechanism. These are determined by developing a model to describe the forces acting on the blades. Finally, the model and its application are discussed in Section 4, followed by the conclusion in Section 5 .

\section{Model Overview}

As described above, the CVP propeller has not been realized for commercial use yet. Therefore, the CVP propeller considered is a modified CP propeller when modelling the loads acting on the CVP propeller. Therefore, the study considers the geometry utilized for a CP propeller and the operating conditions for this CP propeller. The modelling of the loads acting on the CVP propeller blades is therefore associated with the modelling of the loads acting on the CP propeller blades, except that the CVP propeller blades make a cyclic variation of the pitch of the blades, while the CP propeller does not.

The modelling of the loads acting on the CP propeller blades has previously been presented in $[10,13-18]$. Common for $[10,13-18]$ are that the models are based on the rigid body motion of the pitch of the propeller blades and that they account for the loads, due to the hydrodynamic, centrifugal and frictional loads. The model for the hydrodynamics varies for each of the articles [13-17], but the friction modelling is generally based on [15,16]. The studies are either made to analyze the dynamic performance of the CP propeller $[13,17]$, make pitch control for performance improvements [18], or determine the pitch fretting motion [14-16].

The modelling of the CVP propeller considered is based on inverse rigid body motion modelling, since the pitch motion for the CVP propeller is known. Therefore, the models purpose is to determine the required power and torque to pitch the blades according to the desired motion. Newton's second law of motion is used to model the pitching motion of the CVP propeller blades as:

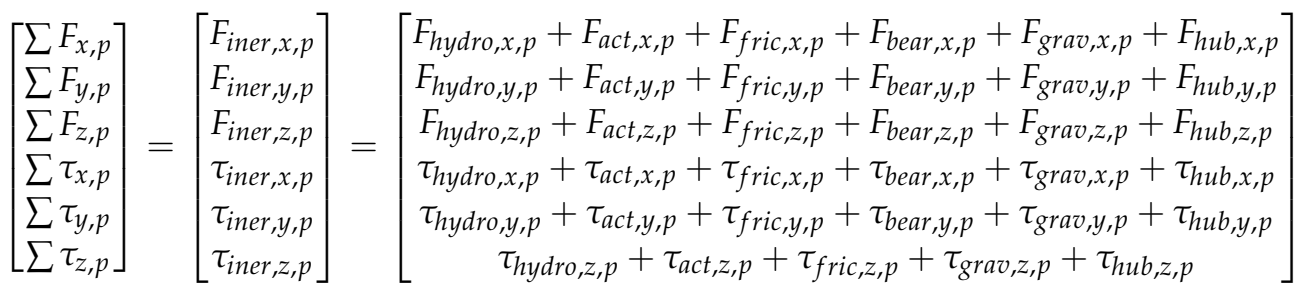

where $\sum F_{i, p}$ and $\sum \tau_{i, p}$ are the sum of forces and torques along/around the $x y z$-axis in the propeller coordinate system, as defined in Section 2.1. The sum of forces and torques are also called the inertial forces and torques, hence noted as $F_{i n e r, i, p}$ and $\tau_{\text {iner,i,p }}$, respectively. The forces and torques in Equation (1) have three indexes in the subscript, where a comma separates each index. The first index indicates the load as either; hydrodynamic, gravitational, hub, friction, bearing or actuator. The second index indicates which of the $x-, y$ or $z$-axis the force/torque is along/about. The third index indicates in which coordinate system the load is defined, which can be either $s, p$ or $b$ for the ship, propeller and blade coordinate system, respectively. This is further elaborated in Section 2.1. The six expressions in Equation (1) each describe a degree of freedom for the propeller blade. The propeller blade is only free to rotate about its spindle/pitch axis (i.e., $z$-axis), which is why there are no bearing loads for the moment about the $z$-axis in Equation (1). The other degrees 
of freedom are locked because the propeller blade is fixed to the propeller hub due to the blade bearing.

The six loads acting on the propeller blade are explained in the following six subsections. They are:

- Hydrodynamic loads: The pressure and viscous effects acting on the propeller blade due to the interaction between the fluid and the propeller.

- Gravitational loads: Due to gravity.

- Hub loads: Resulting from the pressure of the lubrication oil inside the hub.

- Frictional loads: Between the blade foot and hub.

- Bearing loads: Due to the bearing exerting a load on the blade for the blade to follow the motion.

- Actuator loads: Due to the actuator used to control the pitch of the blade.

\subsection{Inertia Loads}

To describe the inertia loads acting on the CVP propeller, different coordinate systems are used. These are shown in Figure 3, along with the definitions used for the pitch trajectory.

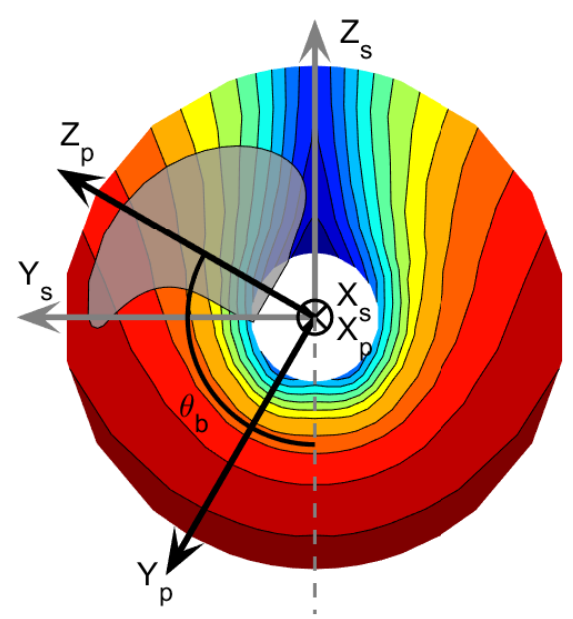

(a)

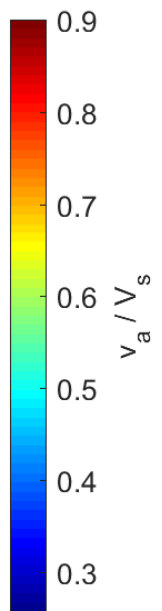

3

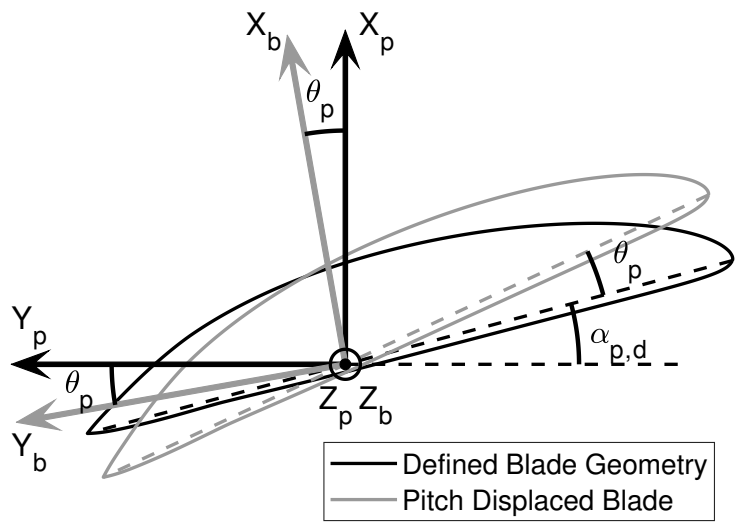

(b)

Figure 3. (a) Ship and propeller coordinate system and definition of the position of the propeller blade in wake field $\theta_{b}$. (b) Propeller and blade coordinate system and definition of the pitch displacement of the propeller blade $\theta_{p}$.

In Figure 3, three coordinate systems are defined, which are the ship, propeller and blade coordinate systems.

- The ship coordinate system $\left(X_{s}, Y_{s}, Z_{s}\right)$ is fixed to the ship with origins in the centre of the propeller, where the $x$-axis extends from the propeller centre towards the ship bow, the $y$-axis is towards the port side, and the z-axis is upward.

- The propeller coordinate system $\left(X_{p}, Y_{p}, Z_{p}\right)$ (one coordinate system for each propeller blade), in which the $z$-axis of the coordinate system is aligned with the blade's spindle axis. The coordinate system rotates with the propeller, and its $x$-axis is aligned with the propeller's $x$-axis, which also aligns with the ship's longitudinal axis.

- The blade coordinate $\left(X_{b}, Y_{b}, Z_{b}\right)$ (one coordinate system for each blade) rotates with the pitching of the propeller blade. The $z$-axis of the blade coordinate system is aligned with the $z$-axis of the propeller coordinate system.

The transformation between each of the coordinate system is made as: 


$$
\left[\begin{array}{l}
X_{p, i} \\
Y_{p, i} \\
Z_{p, i}
\end{array}\right]=\underbrace{\left[\begin{array}{ccc}
1 & 0 & 0 \\
0 & -\cos \left(\theta_{b, i}\right) & -\sin \left(\theta_{b, i}\right) \\
0 & \sin \left(\theta_{b, i}\right) & -\cos \left(\theta_{b, i}\right)
\end{array}\right]}_{T_{s, p}\left(\theta_{b, i}\right)}\left[\begin{array}{c}
X_{S} \\
Y_{S} \\
Z_{s}
\end{array}\right],\left[\begin{array}{c}
X_{b, i} \\
Y_{b, i} \\
Z_{b, i}
\end{array}\right] \quad=\underbrace{\left[\begin{array}{ccc}
\cos \left(\theta_{p, i}\right) & -\sin \left(\theta_{p, i}\right) & 0 \\
\sin \left(\theta_{p, i}\right) & \cos \left(\theta_{p, i}\right) & 0 \\
0 & 0 & 1
\end{array}\right]}_{T_{p, b}\left(\theta_{p, i}\right)}\left[\begin{array}{c}
X_{p, i} \\
Y_{p, i} \\
Z_{p, i}
\end{array}\right]
$$

Here, $\boldsymbol{T}_{s, p}$ is the transformation matrix from the ship to the propeller coordinate system and $\boldsymbol{T}_{p, b}$ is the transformation matrix from the propeller to the blade coordinate system. The transformation matrices $T_{p, s}$ and $T_{b, p}$ are determined as the inverse of the transformation matrices $T_{s, p}$ and $T_{p, b}$, respectively. $\theta_{b, i}$ is the $i$ 'th blades position in the wake field, as shown in Figure 3a. $\theta_{p, i}$ is the $i^{\prime}$ th blades pitch displacement relative to the design pitch of the blade, as shown in Figure $3 \mathrm{~b}$. The pitch of the CVP propeller blades varies cyclically with the blade's position in the wake field. The cyclic varying pitch trajectory $\left(\alpha_{p}\right)$ of the propeller blades is described with a Fourier series as:

$$
\alpha_{p}\left(\theta_{b}\right)=\underbrace{a_{0}}_{\alpha_{p, d}}+\underbrace{\sum_{n=1}^{N} a_{n} \cos \left(n \theta_{b}\right)+b_{n} \sin \left(n \theta_{b}\right)}_{\theta_{p}\left(\theta_{b}\right)}
$$

where $\alpha_{p, d}$ is the pitch of the propeller blade (usually considered at the 0.7 non-dimensional radius of the propeller). $a_{n}$ and $b_{n}$ are the Fourier series coefficients, where $n$ is the harmonics of the Fourier series, with $N$ being the largest harmonic included in the Fourier series.

The inertia loads are determined as the change in the linear and angular momentum of the propeller blade in the inertial reference frame. It is assumed that the ship coordinate system is an inertial reference frame. The ship is therefore assumed to have a constant velocity vector. Furthermore, it is assumed that the propeller blade is rigid.

The inertia force vector $\left(\boldsymbol{F}_{\text {iner }, \mathrm{s}}=\left[\begin{array}{lll}F_{\text {iner }, x, s} & F_{\text {iner }, y, s} & F_{\text {iner }, z, s}\end{array}\right]^{T}\right)$ in the ship coordinate system is equal to the change in the linear momentum vector $\left(G_{S}\right)$ in the ship coordinate system as:

$$
\begin{aligned}
& \boldsymbol{F}_{\text {iner }, \mathrm{s}}=\dot{\boldsymbol{G}}_{s} \rightarrow \boldsymbol{F}_{\text {iner,s}}=m_{b} \dot{\boldsymbol{v}}_{C M, s} \rightarrow \boldsymbol{F}_{\text {iner }, s}=m_{b} \ddot{\boldsymbol{r}}_{C M, s} \rightarrow \\
& \boldsymbol{F}_{\text {iner,s }}=m_{b} \frac{d^{2}}{d t^{2}}\left(\boldsymbol{T}_{p, s} \boldsymbol{T}_{b, p}\right) \boldsymbol{r}_{C M} \rightarrow \boldsymbol{F}_{\text {iner }, s}=m_{b} \underbrace{\left(\ddot{\boldsymbol{T}}_{p, s} \boldsymbol{T}_{b, p}+2 \dot{\boldsymbol{T}}_{p, s} \dot{\boldsymbol{T}}_{b, p}+\boldsymbol{T}_{p, s} \ddot{\boldsymbol{T}}_{b, p}\right)}_{\boldsymbol{T}_{G, s}} \boldsymbol{r}_{C M} \rightarrow \\
& \boldsymbol{F}_{\text {iner }, \mathrm{s}}=m_{b} \boldsymbol{T}_{G, s} \boldsymbol{r}_{C M}
\end{aligned}
$$

where $\boldsymbol{r}_{C M, s}$ and $\boldsymbol{v}_{C M . s}$ are the position and velocity vectors of the propeller blades center of mass in the ship coordinate system. $\boldsymbol{r}_{C M}$ is the position vector of the propeller blades' centre of mass in the blade coordinate system where it is constant. $m_{b}$ is the mass of the propeller blade. To use the inertia force vector in Equation (1), it has to be transformed to the propeller coordinate system as:

$$
\boldsymbol{F}_{\text {iner }, p}=\boldsymbol{T}_{s, p} \boldsymbol{F}_{\text {iner }, \mathrm{s}}=m_{b} \underbrace{\boldsymbol{T}_{s, p} \boldsymbol{T}_{G, s}}_{\boldsymbol{T}_{G, p}} \boldsymbol{r}_{C M}=m_{b} \boldsymbol{T}_{G, p} \boldsymbol{r}_{C M}
$$

The inertia torque vector $\left(\boldsymbol{\tau}_{\text {iner,s }}\right)$ in the ship coordinate system is equal to the change in the angular momentum vector $\left(\boldsymbol{H}_{\text {iner,s }}\right)$ in the ship coordinate system. To make the derivation, the angular velocities vectors in the three coordinate systems are defined as in Equation (6) and the angular momentum vector in each coordinate system is defined as in Equation (7). 


$$
\begin{gathered}
\boldsymbol{\omega}_{p}=\left[\begin{array}{c}
\dot{\theta}_{b} \\
0 \\
\dot{\theta}_{p}
\end{array}\right], \quad \boldsymbol{\omega}_{s}=\boldsymbol{T}_{p, s} \boldsymbol{\omega}_{p}=\left[\begin{array}{c}
\dot{\theta}_{b} \\
\dot{\theta}_{p} \sin \left(\left(\theta_{b}\right)\right) \\
-\dot{\theta}_{p} \cos \left(\left(\theta_{b}\right)\right)
\end{array}\right], \boldsymbol{\omega}_{b}=\boldsymbol{T}_{p, b} \boldsymbol{\omega}_{p} \quad=\left[\begin{array}{c}
\dot{\theta}_{b} \cos \left(\left(\theta_{p}\right)\right) \\
-\dot{\theta}_{b} \sin \left(\left(\theta_{p}\right)\right) \\
-\dot{\theta}_{p}
\end{array}\right] \\
\boldsymbol{H}_{b}=\boldsymbol{I}_{b} \boldsymbol{\omega}_{b} \\
\boldsymbol{H}_{p}=\boldsymbol{I}_{p} \boldsymbol{\omega}_{p} \rightarrow \boldsymbol{T}_{b, p} \boldsymbol{H}_{b}=\boldsymbol{T}_{b, p} \boldsymbol{I}_{b} \boldsymbol{\omega}_{b}=\boldsymbol{T}_{b, p} \boldsymbol{I}_{b} \boldsymbol{T}_{p, b} \boldsymbol{\omega}_{p} \\
\boldsymbol{H}_{s}=\boldsymbol{I}_{s} \boldsymbol{\omega}_{s} \rightarrow \boldsymbol{T}_{p, s} \boldsymbol{H}_{p}=\boldsymbol{T}_{p, s} \boldsymbol{T}_{b, p} \boldsymbol{I}_{b} \boldsymbol{T}_{p, b} \boldsymbol{\omega}_{p}=\boldsymbol{T}_{p, s} \boldsymbol{T}_{b, p} \boldsymbol{I}_{b} \boldsymbol{T}_{p, b} \boldsymbol{T}_{s, p} \boldsymbol{\omega}_{s}
\end{gathered}
$$

The inertia torque vector in the ship coordinate system is determined as:

$$
\begin{aligned}
& \boldsymbol{\tau}_{\text {iner }, s}=\dot{\boldsymbol{H}}_{s} \rightarrow \boldsymbol{\tau}_{\text {iner }, s}=\frac{d}{d t}\left(\boldsymbol{I}_{s} \boldsymbol{\omega}_{s}\right) \rightarrow \boldsymbol{\tau}_{\text {iner }, s}=\frac{d}{d t}\left(\boldsymbol{T}_{p, s} \boldsymbol{T}_{b, p} \boldsymbol{I}_{b} \boldsymbol{T}_{p, b} \boldsymbol{T}_{s, p} \boldsymbol{\omega}_{s}\right) \rightarrow
\end{aligned}
$$

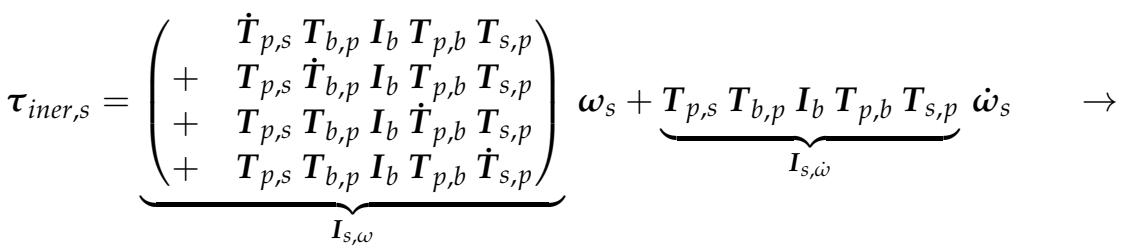

$$
\begin{aligned}
& \tau_{\text {iner }, s}=I_{s, \omega} \omega_{s}+I_{s, \dot{\omega}} \dot{\omega}_{s}
\end{aligned}
$$

For the modelling in Equation (1), it is necessary to have the inertia torques defined in the propeller coordinate system. The inertia torques are therefore transformed to the inertia coordinate system as:

$$
\begin{aligned}
& \boldsymbol{\tau}_{\text {iner }, p}=\boldsymbol{T}_{s, p} \boldsymbol{\tau}_{\text {iner }, p} \rightarrow \boldsymbol{\tau}_{\text {iner }, p}=\boldsymbol{T}_{s, p}\left(\boldsymbol{I}_{s, \omega} \boldsymbol{\omega}_{s}+\boldsymbol{I}_{s, \dot{\omega}} \dot{\boldsymbol{\omega}}_{s}\right) \rightarrow \\
& \boldsymbol{\tau}_{\text {iner }, p}=\underbrace{\boldsymbol{T}_{s, p} \boldsymbol{I}_{s, \omega} \boldsymbol{T}_{p, s}}_{\boldsymbol{I}_{p, \omega}} \boldsymbol{\omega}_{p}+\underbrace{\boldsymbol{T}_{s, p} \boldsymbol{I}_{s, \omega} \boldsymbol{T}_{p, s}}_{\boldsymbol{I}_{p, \omega}} \dot{\boldsymbol{\omega}}_{p} \rightarrow \boldsymbol{\tau}_{\text {iner }, p}=\boldsymbol{I}_{p, \omega} \boldsymbol{\omega}_{p}+\boldsymbol{I}_{p, \dot{\omega}} \dot{\boldsymbol{\omega}}_{p}
\end{aligned}
$$

\subsection{Hydrodynamic Loads}

The determination of the hydrodynamic loads acting on the CVP propeller blades has been studied in $[2,7,12]$. In $[2,12]$, the hydrodynamic loads are determined quasi-statically by interpolating the quasi-steady hydrodynamic loads for a series of propellers with different pitch settings. However, this approach does not account for the hydrodynamic loads' pitch rate and acceleration dependency. Instead, in [7], the hydrodynamic loads are determined using a modified unsteady lifting surface program, which should account for the unsteadiness, due to the pitch, pitch rate and acceleration. In the current paper, the hydrodynamic loads are instead determined by using unsteady-Reynolds-average-NavierStokes (URANS) computational fluid dynamics (CFD). The benefit of using URANS CFD simulations to determine the hydrodynamic loads for the CVP propeller is the ability to account for flows with complex vortex structures and/or viscous effects [19].

\section{Simulation Set-Up}

The URANS CFD simulations of the CVP propeller are made using the commercial program STAR-CCM+12.02.010. The propeller used in the CFD simulations is described in Section 3. The domain for the CFD simulations is cylindrical, as shown in Figure 4. As shown in the figure, the domain consists of a stationary region (enclosing the propeller region) and a rotating propeller region (enclosing the propeller). The rotation of the rotating propeller region is made with a sliding mesh with an interface between the two regions. The cyclic pitching of the propeller blades is made by displacing the blade surface mesh. The rest of the mesh in the rotating propeller region morphs to accompany this displacement. The wall boundaries are set to non-slip surfaces, and the far-field boundary is set to a slip surface. The outlet is set as a pressure outlet with constant pressure, and 
the inlet boundary is set to the velocity field. The domain dimensions for the stationary domain are set to $L_{r}=10.5 D_{p}, L_{b}=12.7 D_{p}$ and $L_{f}=4 D_{p}$, where $D_{p}$ is the propeller diameter. The domain dimensions $L_{r}$ and $L_{b}$ are determined through a domain study using a quasi-steady simulation setup. The inlet velocity field is the radial varying wake field of the ship when using the $0^{\prime}$ th harmonics of radial varying Fourier series analysis of the wake field velocities. The uncertainty in the hydrodynamic loads is included in the uncertainty analysis of the simulation results. The domain dimension $L_{f}$ is determined through a wake field study, where the error in the wake field velocity components at the propeller is minimized. According to the tank test report, the axial velocity components of the measured wake field are scaled by the ratio between the effective wake coefficient and the nominal wake coefficient. This scaled axial velocity field is imposed on the inlet of the simulation. This measured wake field does not cover the entire domain; it is therefore extrapolated to cover the full domain. The extrapolation towards the shaft is made, so the velocity at the shaft is zero due to the non-slip condition. The extrapolation towards the radial boundary is made linearly for each circumferential position based on the slope of the two outermost measurements of the wake field and limited to the ships speed. To include the transverse velocity components in the simulation, momentum sources are included in the area between the propeller and the inlet. The momentum sources are determined by scaling the transverse velocity components by a gain. The gain is determined iteratively through several simulations without the propeller blades, as in [20].

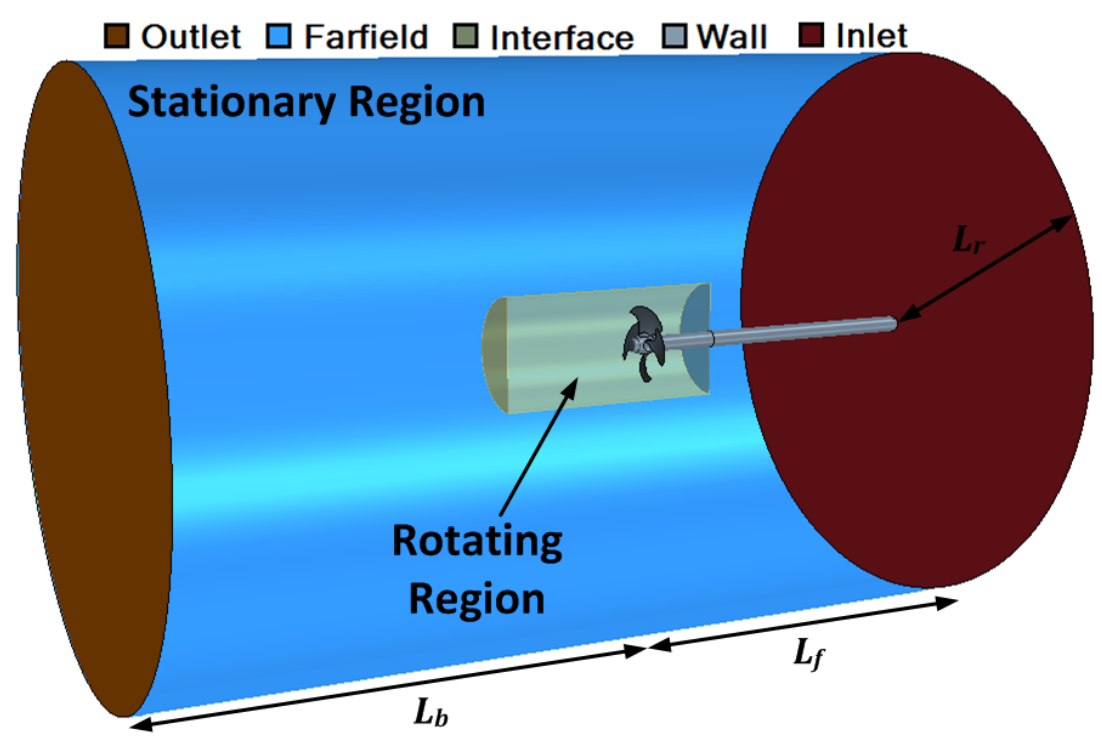

Figure 4. The domain used in the CFD simulations of the WP propeller in a non-uniform wake field.

The URANS CFD simulations of the CVP propeller are made as unsteady single-phase simulations, assuming an incompressible fluid and using the segregated flow solver. The discretization order for both spatial and temporal discretization is second order. Furthermore, the simulations include gravity to account for the hydrostatic pressure. The turbulence is modelled using the SST $\mathrm{k} \omega$ RANS model without the transition model, because the simulations are made using the full-scale propeller. A description of the discretization error is found in Appendix A. 


\subsection{Gravitational Loads}

The gravitational force acting on the propeller blades is in the negative z-direction in the ship coordinate system. Therefore, to be applicable in Equation (1), it is transformed to the propeller coordinate system as:

$$
\boldsymbol{F}_{\text {grav }, s}=\left[\begin{array}{c}
0 \\
0 \\
-m_{b} g
\end{array}\right] \rightarrow \boldsymbol{F}_{\text {grav }, p}=\boldsymbol{T}_{s, p} \boldsymbol{F}_{\text {grav }, s}
$$

where $g$ is the gravitational acceleration. To determine the torque components due to gravity, the cross product of the gravitational force vector and the position vector of the centre of mass for the propeller blade is taken and transformed to the propeller coordinate system as:

$$
\begin{aligned}
& \boldsymbol{\tau}_{\text {grav }, s}=\boldsymbol{F}_{\text {grav }, s} \times r_{C M, s} \rightarrow \tau_{\text {grav }, s}=\boldsymbol{F}_{\text {grav }, s} \times \boldsymbol{T}_{p, s} \boldsymbol{T}_{b, p} \boldsymbol{r}_{C M} \\
& \boldsymbol{\tau}_{\text {grav }, p}=\boldsymbol{T}_{s, p} \boldsymbol{\tau}_{\text {grav }, s} \rightarrow \boldsymbol{\tau}_{\text {grav }, p}=\boldsymbol{T}_{s, p}\left(\boldsymbol{F}_{\text {grav }, s} \times \boldsymbol{T}_{p, s} \boldsymbol{T}_{b, p} \boldsymbol{r}_{C M}\right)
\end{aligned}
$$

\subsection{Hub Loads}

The hub load acting on the propeller blade is due to the lubrication oil pressure inside the hub. The lubrication oil is used to lubricate the blade bearing. The lubrication oil is typically pressurized by elevating the tank with the lubrication oil a couple of meters above the waterline. In the propeller coordinate system, this gives a force contribution in the z-direction, which is determined as:

$$
\boldsymbol{F}_{h u b, p}=\left[\begin{array}{c}
0 \\
0 \\
p_{h u b} A_{b f}
\end{array}\right]
$$

where,

$$
p_{\text {hub }}=\rho_{\text {oil }} g\left(\left(h_{s}+h_{\text {tank }}\right)+h_{h b} \cos \left(\theta_{b}\right)\right)
$$

$p_{h u b}$ is the pressure of the lubrication oil inside the hub acting on the bottom of the blade foot. $A_{b f}$ is the area of the bottom of the blade foot. $\rho_{\text {oil }}$ is the density of the lubrication oil. $h_{s}$ is the submersion depth of the propeller shaft line. $h_{\text {tank }}$ is the evaluation of the lubrication tank above the waterline, and $h_{h b}$ is the distance from the hub centre to the bottom of the blade foot, cf. Figure 5 .

\subsection{Frictional Loads}

Most measurements of the friction in $\mathrm{CP}$ propellers are based on measuring the pressure difference over the servo piston during changes in the pitch, as shown in $[10,21]$. These measurements include the friction contribution for all the components throughout the $\mathrm{CP}$ propeller system. Modelling of the friction between the hub and the propeller blade has been made in [13-17]. The modelling approach used in this paper for the friction between the hub and propeller blade is one proposed by the current authors. The approach models the friction loads as static Coulomb friction and does not account for Stribeck or viscous friction components. This assumption seems reasonable, since the peripheral velocity is limited, whereby the contribution from the viscous effect is also limited. However, the available data for the friction coefficients in the blade bearing are limited and uncertain. Therefore, further research has to be done to determine these coefficients accurately. A conservative estimate of the Coulomb friction coefficient is used to partially account for the Stribeck and viscous friction components. This coefficient is 50\% larger than the coefficient 
determined in [15], which should cover the potential contributions from the Stribeck and viscous friction. The frictional loads are determined as:

$$
\left[\begin{array}{l}
\boldsymbol{F}_{f r i c, b b} \\
\boldsymbol{\tau}_{f r i c, b b}
\end{array}\right]=\left\{\begin{array}{cc}
{\left[\begin{array}{l}
\boldsymbol{F}_{c, b b} \\
\boldsymbol{\tau}_{c, b b}
\end{array}\right] \operatorname{sign}\left(\dot{\theta}_{p}\right)} & \text { if } \dot{\theta}_{p} \neq 0 \\
\kappa\left[\begin{array}{l}
\boldsymbol{F}_{c, b b} \\
\boldsymbol{\tau}_{c, b b}
\end{array}\right] \operatorname{sign}\left(\tau_{e x t, z, b b}\right) & \text { if } \dot{\theta}_{p}=0
\end{array}\right.
$$

where,

$$
\begin{aligned}
\kappa & =\min \left(\left|\frac{\tau_{\text {ext }, z, b b}}{\tau_{c, z, b b}}\right|, 1\right) \\
\tau_{\text {ext }, z, b b} & =\tau_{\text {hydro }, z, b b}+\tau_{a c t, z, b b}+\tau_{\text {grav }, z, b b}+\tau_{h u b, z, b b}
\end{aligned}
$$

$\boldsymbol{F}_{f r i c, b b}$ and $\boldsymbol{\tau}_{f r i c, b b}$ are the frictional force and torque in the blade bearing coordinate system, see Figure 5. $\boldsymbol{F}_{c, b b}$ and $\boldsymbol{\tau}_{c, b b}$ are the Coulomb friction force and torque in the blade bearing coordinate system, which depends on the bearing loads, Coulomb friction coefficient and the load distribution assumption. $\kappa$ is a scaling component to account for the friction when the pitch rate is zero. $\tau_{e x t, z, b b}$ is the torque used for exciting the pitch motion of the propeller blade. The friction model can be extended to include the viscous friction by adding $B_{v} \dot{\theta}_{p}$ to Equation (15) where $B_{v}$ is the viscous friction coefficient.

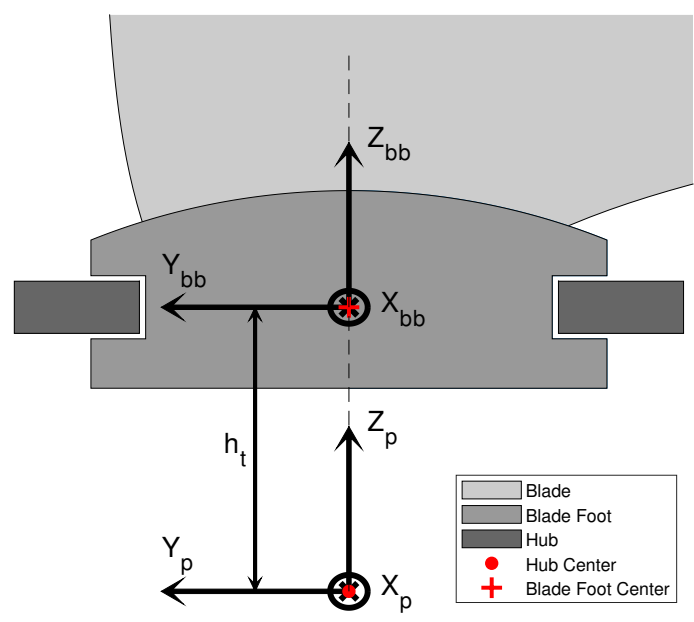

Figure 5. Blade bearing coordinate system.

The blade bearing coordinate system is used to model the friction to get the appropriate contact loads in the bearing. The coordinate system is a translation of the propeller coordinate system along the spindle axis to the centre of the bearing, as shown in Figure 5 . It is assumed that the blade centre bearing is also the centre of stiffness, about which the blade will tilt due to the $x$ - and $y$-axis torques. The forces in the blade bearing coordinate system are the same as in the propeller coordinate system, where the torque vector $\left(\boldsymbol{\tau}_{b b}\right)$ in the blade bearing coordinate system is determined as:

$$
\tau_{b b}=\tau_{p}-\boldsymbol{r}_{t} \times \boldsymbol{F}_{p}
$$

$\boldsymbol{\tau}_{p}$ and $\boldsymbol{F}_{p}$ are the torque and force vector in the propeller coordinate system. $\boldsymbol{r}_{t}$ is the translation vector from the propeller coordinate system to the blade bearing coordinate system.

The Coulomb friction loads $\left(\boldsymbol{F}_{c, b b}\right.$ and $\left.\boldsymbol{M}_{c, b b}\right)$ are determined assuming that the bearing force $F_{b e a r, z, b b}$ acts uniformly on the axial surfaces of the bearing (i.e., the surface perpendicular to the spindle axis). The bearing forces $F_{b e a r, x, b b}$ and $F_{b e a r, x, b b}$ are assumed to act on the radial surface of the bearing with an elliptical load distribution according to the theory of 
Hertzian contact mechanics. The bearing torques $\tau_{b e a r, x, b b}$ and $\tau_{b e a r, x, b b}$ are assumed to be distributed on both the axial and radial surfaces of the bearing according to the distribution ratio $(D R)$ :

$$
D R=\frac{2 R_{b b}-h_{b b}}{2 R_{b b}}
$$

$R_{b b}$ is the outer radius of the blade bearing, and $h_{b b}$ is the height of the blade bearing. The bearing torque acting on the axial surfaces is $D R \tau_{b e a r, x y, b b}$ and the bearing torque acting on the radial surface is $(1-D R) \tau_{\text {bear }, x y, b b}$. The bearing torque in the radial surface is modelled according to Hertzian contact mechanics. The bearing torque in the axial surfaces is modelled to be varying linearly in the radial direction and sinusoidally along the circumference.

\subsection{Actuator Loads}

The modelling of the actuator loads depends on the topological design of the pitch mechanism. However, since an actual CVP propeller does not exist, the actuator loads cannot be modelled according to a specific design. The modelling of the actuator loads is therefore made as a pure pitching torque contribution as:

$$
\boldsymbol{F}_{a c t, p}=\mathbf{0}, \quad \boldsymbol{\tau}_{a c t, p}=\left[\begin{array}{lll}
0 & 0 & \tau_{a c t, z, p}
\end{array}\right]^{T}
$$

The actuator forces are zero, and actuator torques consist of only a torque around the spindle/pitch axis. This actuator model is equivalent to a motor being mounted on each propeller blade inside the hub. If other actuator topologies are to be investigated, they must be modelled accordingly and account for the losses due to friction. The modelling of the actuator loads in Equation (20) is therefore assessed to be the minimum required actuator loads to pitch the CVP propeller blades according to the desired pitch trajectories.

\subsection{Bearing Loads}

The loads from the bearings on the blade are not determined explicitly as the previously described loads. Instead, the bearing loads are determined to satisfy the force and torque balance in Equation (1). Because the frictional load depends on the bearing loads, the bearing loads have to be determined iteratively for each blade position in the wake field. The iterative solving procedure is (Algorithm 1):

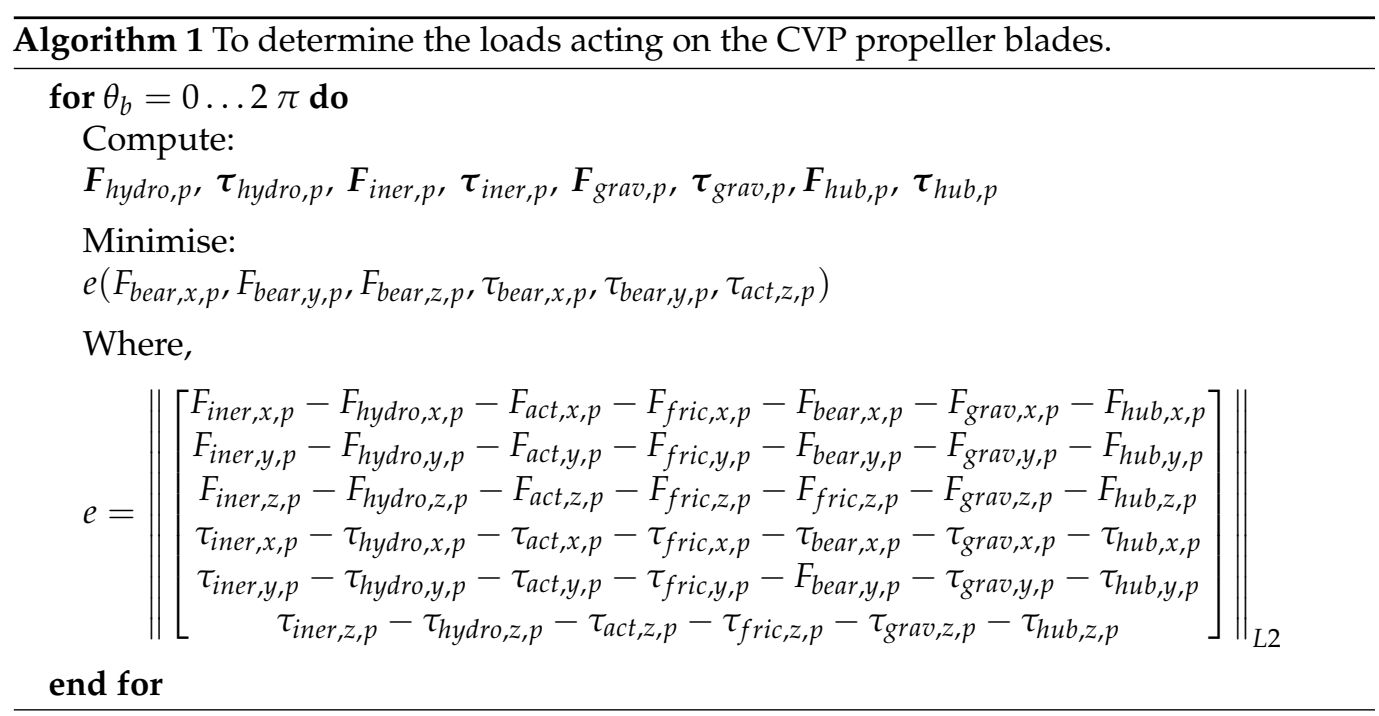

The above algorithm computes the hydrodynamic-, inertia-, gravitational- and hub loads using the methods described in the previous sections for each blade position. The 
bearing loads and the actuation spindle torque $\left(\tau_{a c t, z, p}\right)$ are determined through minimizing the Euclidean-norm of the error of the system of equations in Equation (1).

\section{CVP Propeller Model Application}

To apply the model, the propeller considered in $[8,11,12]$ is used. In [8], three pitch trajectories are determined for the CVP propeller using a quasi-steady analysis method. The Fourier series approximations of pitch displacement, pitch rate and pitch accelerations for these pitch trajectories are shown in Figure 6. The blade position in Figure 6 is the blade's position in the wake field, as defined in Figure 3a. Therefore, the blade is in the low-velocity part of the wake field around a blade position of $180^{\circ}$. In [8], the three pitch trajectories; "Constant", "Cosine" and "Variable" are determined. The variable pitch trajectory is determined iteratively using a quasi-steady lifting line approach, whereby variation in the blade thrust and transient cavitation is almost eliminated while maximizing the efficiency. The cosine pitch trajectory is the best pitch trajectory containing a single first-order harmonic, which reduces the variations in the blade thrust and maximizes the efficiency relative to the constant pitch trajectory. The constant pitch trajectory is equivalent to the operation of the CP propeller. Furthermore, in [8], the geometry of the propeller blade is adapted for each of the pitch trajectories to obtain optimum performance of the propeller. However, the current paper uses the propeller blades' original geometry for all three pitch trajectories. Therefore, the difference in the loads is only due to the pitch trajectory.
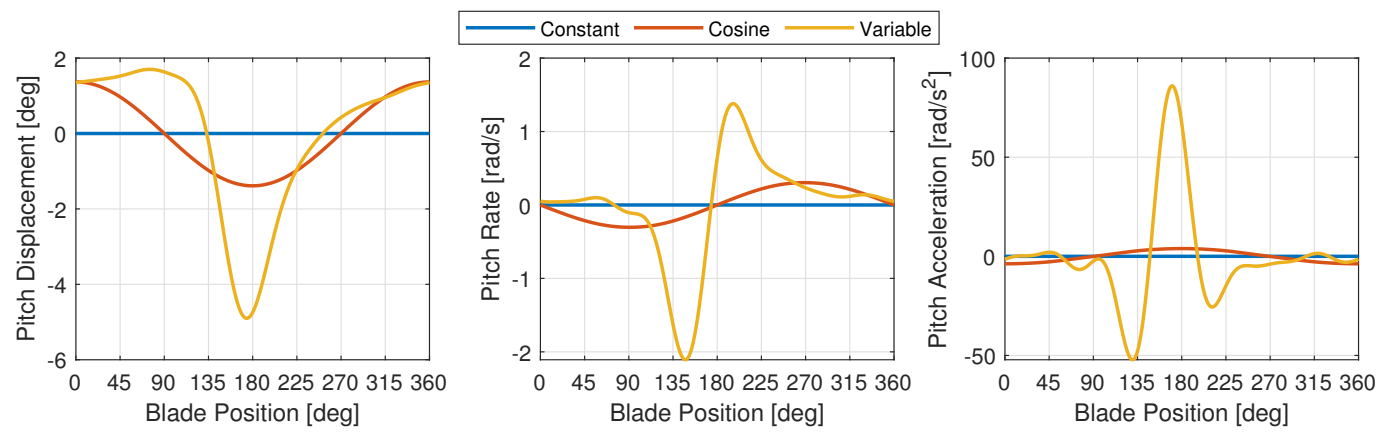

Figure 6. Pitch displacement, pitch rate and pitch acceleration of the pitch trajectories determined in [8].

The propeller design parameters and the parameters used for the modelling of the CVP propeller are listed in Table A1 in Appendix B. The inertia, gravitational and hub loads are determined using the parameters in Table A1, and the modelling approaches described in Section 2 for each of the pitch trajectory in Figure 6. The hydrodynamic loads and their uncertainty are determined using the simulation approach described in Section 2 for each of the pitch trajectories. These loads are shown in Figure 7.

From Figure 7, it is seen that for the constant pitch trajectory, the hydrodynamic loads change significantly around the wake peak at $180^{\circ}$. The change in the hydrodynamic loads for the cosine pitch trajectory is generally reduced, which is expected from the results in [8]. Finally, it is seen that the hydrodynamic loads for the variable pitch trajectory do not match with the expectation from [8]. The variation in the hydrodynamic loads is here not reduced further than for the cosine pitch trajectory. Instead, the variation in the hydrodynamic loads is significantly increased for the variable pitch trajectory. This is likely due to the quasi-steady approach used to determine the pitch trajectories, which does not account for the dynamic effects in the hydrodynamic response. It, therefore, does not seem appropriate to use the quasi-steady approach to determine the pitch trajectory that should minimize the variation in the hydrodynamic loads. Furthermore, the uncertainty of the URANS CFD simulations is generally the largest when the propeller blade is at the wake peak. 

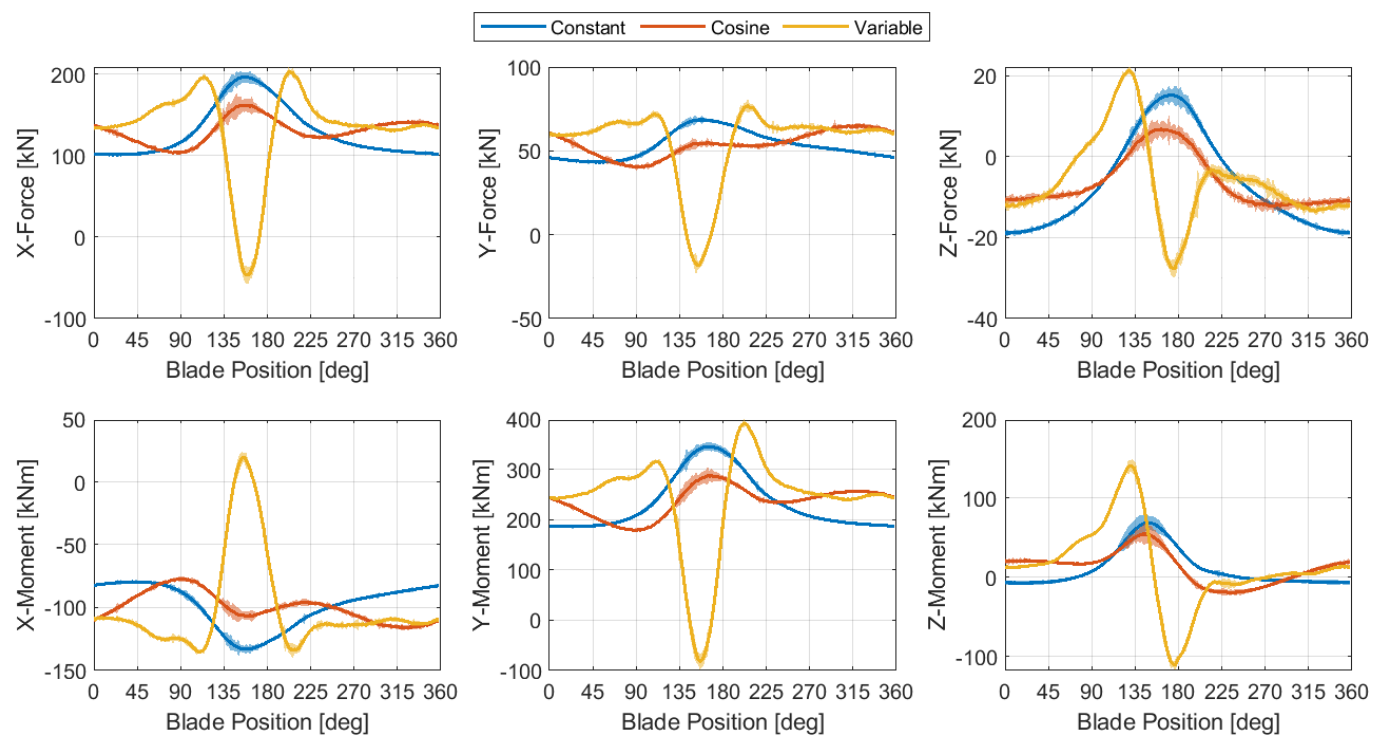

Figure 7. The hydrodynamic loads acting on the CVP propeller blades for each of the three pitch trajectories determine URANS CFD simulations.

The bearing, frictional and actuator loads are determined by using Algorithm 1 and are determined for each of the three pitch trajectories. The determined torques about the spindle axis are shown in Figure 8 for each of the pitch trajectories.

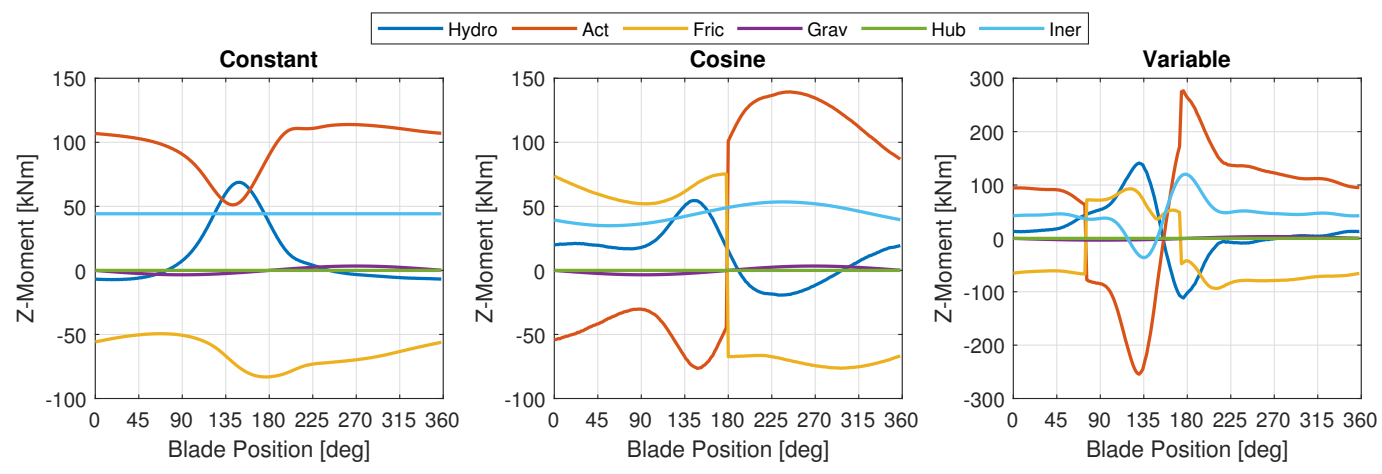

Figure 8. Determined torque components for the propeller blade to pitch according to the pitch trajectories. The results for each pitch trajectory are shown in each of the plots according to their title.

From Figure 8, we can see that the main contributors to the actuator torque are the inertia, hydrodynamic and frictional torques. However, this does not imply that the loads due to gravity and the lubrication oil may be neglected, as they may influence the required actuator torque through the frictional load, which is again due to the frictional dependency on the bearing loads. To assess whether these loads may be neglected may require a sensitivity study.

The requirements for the actuator are made concerning the required actuator power and torque. The actuator power $\left(P_{a c t}\right)$ is determined as:

$$
P_{a c t}\left(\theta_{b}\right)=\tau_{a c t, z, p}\left(\theta_{b}\right) \dot{\theta}_{p}\left(\theta_{b}\right)
$$

The actuator power and torque include the uncertainty of the URANS CFD simulations, which are shown in Figure 9, with the average, minimum and maximum values given in Table 1. 


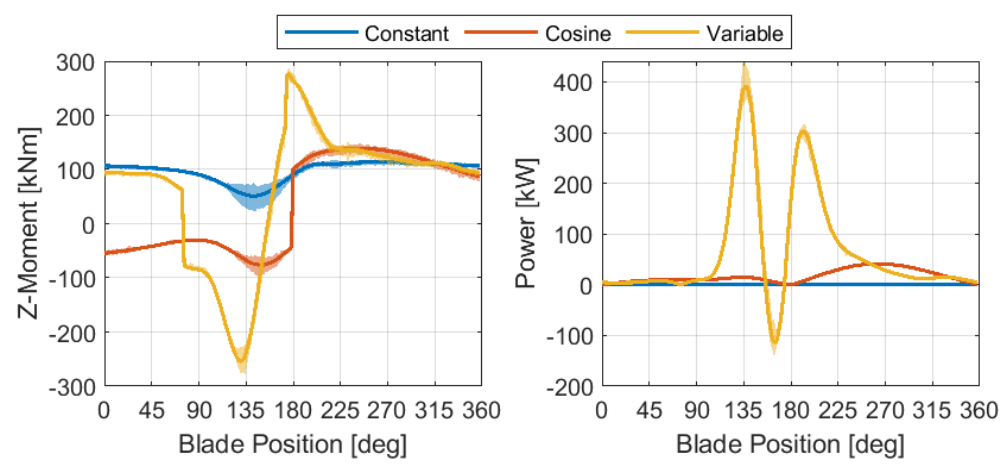

Figure 9. Required actuator torque and power with their uncertainties due to the uncertainty in the hydrodynamic loads for each of the three pitch trajectories.

Table 1. Average, maximum and minimum actuator torque and power, along with the uncertainties resulting from the uncertainty in the hydrodynamic loads for each of the pitch trajectories.

\begin{tabular}{crcc}
\hline Pitch Trajectory & Constant & Cosine & Variable \\
\hline $\begin{array}{l}\text { Average Actuator } \\
\text { Torque [kNm] }\end{array}$ & $97.6_{-7.7}^{+5.6}$ & $36.6_{-7.4}^{+5.8}$ & $63.9_{-10.0}^{+7.3}$ \\
\hline $\begin{array}{l}\text { Maximum Actuator } \\
\text { Torque [kNm] }\end{array}$ & $113.9_{-4.7}^{+4.6}$ & $139.4_{-6.6}^{+8.7}$ & $276.5_{-6.4}^{+10.8}$ \\
\hline $\begin{array}{l}\text { Minimum Actuator } \\
\text { Torque [kNm] }\end{array}$ & $51.2_{-27.8}^{+17.5}$ & $-76.5_{-21.1}^{+13.8}$ & $-254.5_{-24.7}^{+23.3}$ \\
\hline Average Power [kW] & 0.0 & $16.7_{-1.4}^{+1.1}$ & $63.7_{-6.2}^{+7.4}$ \\
\hline Maximum Power [kW] & 0.0 & $41.3_{-2.2}^{+2.0}$ & $392.3_{-23.2}^{+48.7}$ \\
\hline Minimum Power [kW] & 0.0 & $0.0_{-0.0}^{+0.0}$ & $-113.5_{-28.1}^{+24.6}$ \\
\hline
\end{tabular}

From Figure 9 and Table 1, we can see that the required actuator peak torque is the smallest for the constant pitch trajectory followed by the cosine pitch trajectory. In contrast, the variable pitch trajectory requires the highest peak actuator torque. Therefore, the size of the actuator system has to be the largest for the CVP propeller with the variable pitch trajectory. Therefore, depending on the space available in the propeller hub, it may be necessary to compromise on the CVP propeller's performance to fit the actuator system inside the propeller.

The variable pitch trajectory is the pitch trajectory that requires the most power, both on average and in peak conditions, as shown in Figure 9 and Table 1. Furthermore, the minimum required actuator power with the variable pitch trajectory is negative. This gives the possibility to regenerate some of the power used. In comparison, the average power requirement for the variable pitch trajectory is approximately four times the average power for the cosine pitch trajectory. For this to be beneficial, the propeller efficiency improvement should be larger when using the variable pitch trajectory than when using the cosine pitch trajectory to overcome the extra power required for the variable pitch trajectory for the CVP propeller. Otherwise, the overall propulsion efficiency will go down with the CVP propeller. However, even in this case, it may sometimes still be desirable to use the CVP propeller if other performance metrics like cavitation, pressure pulses, vibrations, and noise are important for a given application. 
For the considered application, the propeller efficiency $\left(\eta_{p}\right)$ and the resulting propeller efficiency $\left(\eta_{r, p}\right)$, which includes the power consumption of the actuator, is determined as:

$$
\begin{aligned}
\eta_{p} & =\frac{\bar{F}_{h y d r o, x, p} V_{a}}{\dot{\theta}_{b} \bar{\tau}_{h y d r o, x, p}} \\
\eta_{r, p} & =\frac{\bar{F}_{h y d r o, x, p} V_{a}}{\dot{\theta}_{b} \bar{\tau}_{h y d r o, x, p}+\bar{P}_{a c t}\left(\theta_{b}\right)}
\end{aligned}
$$

where $\bar{F}_{\text {hydro,x,p }}$ is the cyclic average propeller thrust, $V_{a}$ is the average velocity of the water into the propeller, $\dot{\theta}_{b}$ is the rotational speed of the blade, and $\bar{\tau}_{h y d r o, x, p}$ is the cyclic average propeller torque. The propeller and resulting propeller efficiency for each of the pitch trajectories are shown in Table 2.

Table 2. Propeller efficiency, resulting propeller efficiency and their uncertainty for each pitch trajectory used in the CFD simulations of the CVP propeller. The percentage number in parenthesis is the relative uncertainty.

\begin{tabular}{cccc}
\hline & Constant & Cosine & Variable \\
\hline Propeller Efficiency & $0.647 \pm 0.015( \pm 2.31 \%)$ & $0.647 \pm 0.017( \pm 2.62 \%)$ & $0.637 \pm 0.021( \pm 3.30 \%)$ \\
\hline \multirow{2}{*}{ Resulting Propeller Efficiency } & $\begin{array}{c}0.647 \pm 0.211 \pm 0.085 \\
( \pm 32.60 \% \pm 13.16 \%)\end{array}$ & $\begin{array}{c}0.639 \pm 0.297 \pm 0.113 \\
( \pm 46.49 \% \pm 17.73 \%)\end{array}$ & $\begin{array}{c}0.604 \pm 0.332 \pm 0.118 \\
( \pm 55.00 \% \pm 19.55 \%)\end{array}$ \\
\hline
\end{tabular}

In Table 2, one should note that propeller efficiency in the top row only includes the uncertainties in the CFD calculations, due to the force in the $\mathrm{x}$-direction and torque around the $x$-axis. In contrast, the second row showing the resulting propeller efficiency is based on the forces and torques in all directions, including the much higher resulting uncertainty. The results may be compared by looking at the propeller efficiency and resulting propeller efficiency for the constant trajectory, which is the same. Still, the uncertainties are much higher when including the force and torque uncertainties in all directions. Considering the propeller efficiency, the variation in efficiency is small between each pitch trajectory and within the uncertainties in the calculations. Hence, there is only minimal variation in the obtained thrust relative to the propeller torque with the different trajectories. However, when including the actuator's power required, the resulting propeller efficiency of the variable pitch trajectory drops by roughly $4 \%$ with the used propeller. At the same time, the uncertainty in the calculations is significantly higher, as it includes the calculation of the forces and torques in all directions. However, the above results indicate that an efficiency improvement is not obtained for the considered case, as expected from the literature. This may be because the propeller blade geometry is not reconsidered for each of the cyclic varying pitch trajectories, as done in [8]. However, the latter also builds on models assumptions, which may prove inaccurate.

Furthermore, the variable trajectory used is not optimized for efficiency alone. Instead, it is a compromise for the different radial sections of the blade and the performance metrics, i.e., efficiency, cavitation, and shaft vibrations. Thus, keeping a constant or relatively constant angle of attack may aid the reduction of vibrations and transient cavitation, but at the potential cost of reduced propeller efficiency, as the best propulsion efficiency of a propeller comes from the least propeller wake kinetic energy. However, this requires further studies outside the current scope, but for which the present work will be beneficial. However, with the presented case and assumptions regarding an unaltered propeller design, the considered CVP propeller cannot improve propeller efficiency. Therefore, future investigations have to clarify if adapting the propeller blade geometry with the cyclic varying pitch may improve propulsion efficiency, while also including the power consumption of the cyclic pitch motion. 


\section{Discussion}

In the above, a general model for determining the actuator requirements for the CVP propeller has been presented, and results for a given operating condition (fixed pitch, cosine and variable) have been presented. However, for the design of the actuator system for a CVP propeller, the whole range of operating conditions needs to be considered, as otherwise, the CVP propeller may not function as desired in all operating conditions.

However, the model itself, which has been presented, is general, and is not limited to determining the requirements for a CVP propeller. It may also be used to, e.g., determine the requirements for the actuator system for $\mathrm{CP}$ propellers. It should, in this regard, be noted that the size of the actuator system used in CP propellers is primarily determined through experience. Therefore, if the model may accurately determine the requirements for the actuator system, then it may also be used to reduce the size of the actuator system and thereby increase the propeller's efficiency due to the reduced hub diameter. However, the model's accuracy has to be determined through future experiments, and experimentally validating the model within the required accuracy is in no way straightforward.

Because the model is based on inverse rigid body motion and not rigid body motion, it is unsuitable for determining the blade motion, e.g., control purposes or to predict fretting motion. If the model needs to be used for these purposes, two aspects must be reconsidered to address this issue. One is the discontinuity of the static friction model used, and the other is solving the CFD simulation. The discontinuity of the static friction model may relatively easily be addressed by using a dynamic friction model, as described in [16]. On the other hand, solving the CFD simulation may require extra work. The simulation either has to be solved online as the rigid body motion is solved, or as an alternative, a modelling approach has to be used, which determines the hydrodynamic loads explicitly. To determine actuator requirements for a propeller, the model is, however, acceptable as it is.

\section{Conclusions}

In this paper, a general model for the cyclic varying pitch propeller was presented, which described all the forces and torques working on the propeller. This model may be used to determine the actuator requirements for the propeller. As opposed to previous research, the model presented here is a complete model that benefits from including friction and all inertia effects and incorporating a complete hydrodynamic model—effects that significantly influence the propeller loading.

To use the model, it is necessary to know the propeller geometry, cyclic pitch trajectory and operation conditions. The model accounts for inertia-, hydrodynamic-, hydrostatic-, gravitational-, actuator-, bearing- and frictional forces and torque. Of these, the inertia, gravitational and partly the hydrostatic effects are accounted for explicitly. The hydrodynamic and partly hydrostatic effects are determined through a series of URANS CFD simulations. These also determine the uncertainty of the model, due to discretization and a finite domain size. Finally, the bearing, actuator and frictional forces are determined through an iterative algorithm.

The model has been applied on a propeller previously considered to hereby study the model and its validity. As no data exist for an optimized propeller or pitch trajectory, a single propeller blade geometry has been evaluated with three different pitch trajectories and one operating condition. The results show that the required average power, peak power, and torque increase as the variations in the pitch trajectories increases with the considered propeller. However, these results are influenced by using a CP propeller, as no suited or optimized CVP design and trajectory exist. The results are therefore mainly included to show the validity of the presented method. Therefore, the actuator system size also increases for the example system as the cyclic variation in the pitch increases. With the considered case, the results show that with the given propeller design used, the benefit of a cyclic varying pitch propeller is limited. Still, the variations are within the uncertainty of the model, but do indicate that an energy efficiency improvement is not obtained with the given propeller. However, the results would probably change if a proper CVP propeller 
design was available. The overall efficiency improvement will, in this connection, be a result of the potentially improved propulsion efficiency and the power required for pitching the propeller blades. However, these considerations do not influence the presented model, which is not necessarily limited to the CVP propeller but may also be used to describe, e.g., a CP propeller in cases where the trajectory is known.

Author Contributions: Conceptualization, U.S.F., T.O.A. and J.R.N.; Methodology, U.S.F.; Software, U.S.F.; Validation, U.S.F.; Formal analysis, U.S.F.; Investigation, U.S.F.; Resources, U.S.F. and J.R.N.; Data curation, U.S.F. and H.C.P.; Writing-original draft preparation, U.S.F. and H.C.P.; Writingreview and editing, U.S.F., T.O.A., J.R.N. and H.C.P.; Visualization, U.S.F.; Supervision, T.O.A. and J.R.N.; Project administration, U.S.F.; Funding acquisition, T.O.A., J.R.N. and U.S.F. All authors have read and agreed to the published version of the manuscript.

Funding: This research was partially funded by Innovation Fund Denmark grant number 5016-0017B.

Conflicts of Interest: The authors declare no conflict of interest.

\section{Abbreviations}

The following abbreviations are used in this manuscript:

$\begin{array}{ll}\text { AoA } & \text { Angle of attack } \\ \text { CFD } & \text { Computational fluid dynamics } \\ \text { CP } & \text { Controllable pitch } \\ \text { CVP } & \text { Cyclic varying pitch } \\ \text { FP } & \text { Fixed pitch } \\ \text { URANS } & \text { Unsteady Reynolds average Navier-Stokes }\end{array}$

\section{Appendix A. Discretization Error}

The spatial discretization of the domain is made using STAR-CCM+'s unstructured polyhedral mesh with prism layer cells near the walls. The prism layer cell height is made, such that the average $y+$-value is about 100 for the first prism layer based on the boundary layer development of a flat plate. Because the viscous sublayer is not resolved, STAR-CCM+'s all $y+$ wall function is used. The simulations are made with four to five different spatial discretizations and three to four different temporal discretisations to determine the discretization uncertainty due to the spatial and temporal discretization. The spatial discretization varies from $\approx 1.5$ million cells to $\approx 13.6$ million cells. The chosen temporal discretization depends on the propeller's rotational speed such that the propeller's displacements for a one-time step become $1^{\circ}, 2^{\circ}, 4^{\circ}$ and $8^{\circ}$. Selecting these temporal discretizations resulting in the same blade positions were evaluated for each revolution With the considered propeller speed, this temporal discretization corresponds to time steps of $\approx 1.38 \mathrm{~ms}, \approx 2.75 \mathrm{~ms}, \approx 5.51 \mathrm{~ms}$ and $\approx 11.01 \mathrm{~ms}$, respectively. This should be seen in relation to the maximum pitch rate and pitch acceleration of approximately $2 \mathrm{rad} / \mathrm{s}$ and $85 \mathrm{rad} / \mathrm{s}^{2}$ respectively for the variable pitch trajectory. The former, corresponding to a maximum pitch change of $\approx 0.17^{\circ}$ per time steps, which ensures that convergence criteria, are met as discussed below. The simulations with the finest spatial discretizations are made for all the different temporal discretizations, while the others are only made with the most refined temporal discretization. The discretization error is estimated for each time instance for the roughest temporal discretization, because all the different temporal discretizations are evaluated at these instances. The discretization error for the unsteady simulations is approximated as [22]:

$$
\epsilon_{\phi_{i}} \approx \delta_{\phi_{i}}=\phi_{i}-\phi_{0}=\alpha_{t} h_{t, i}^{p_{t}}+\alpha_{s} h_{s, i}^{p_{x}}
$$

$\epsilon_{\phi_{i}}$ is the discretization error of the $i^{\prime}$ th simulation value, and $\delta_{\phi_{i}}$ is the approximation of it. $\phi_{i}$ is the value/values of interest for the $i^{\prime}$ th simulation, which, in this case, are the hydrodynamic loads. $\phi_{0}$ is the actual value of the hydrodynamic loads. $\alpha_{t}$ and $\alpha_{s}$ are the discretization error gains for the temporal and spatial discretization, respectively. $p_{t}$ and $p_{s}$ 
are the observed orders of temporal and spatial convergences. $h_{t, i}$ and $h_{s, i}$ are the temporal and spatial discretization metrics for the $i^{\prime}$ th simulation, which are determined as:

$$
h_{t, i}=\frac{\Delta t_{i}}{\Delta t_{1}} h_{t, 1} \quad, \quad h_{s, i}=h_{s, 1} \sqrt[3]{\frac{N_{\text {cells }, 1}}{N_{\text {cells }, i}}}
$$

$\Delta t_{i}$ is the $i^{\prime}$ th simulation time step, and a subscript of 1 is for the finest refinement. $N_{\text {cells }, i}$ is the number of cells in the $i^{\prime}$ th simulation, and the subscript of 1 is for the finest refinement. $h_{t, 1}$ and $h_{s, 1}$ are the metrics for the finest refinement of the temporal and spatial discretization, which are both 1 .

The discretization error in Equation (A1) is approximated through a least square estimation for the parameters $\phi_{0}, \alpha_{t}, \alpha_{s}, p_{t}$ and $p_{s}$. If the estimated order of convergence is unrealistic according to the discretization scheme chosen, alternative formulations of the discretization error are determined instead. These alternative formulations are firstand second-order polynomials of the temporal and spatial discretization errors. The estimated discretization error with the lowest standard deviation is used to determine the discretization uncertainty. This approach is similar to the one presented in [22]. From the approximated discretization error, the discretization uncertainty is determined as:

$$
U_{\text {disc }}\left(\phi_{i}\right)= \begin{cases}1.25\left|\epsilon_{\phi_{i}}\right|+2 \sigma & \text { for } \sigma<\Delta_{\phi} \\ 3 \frac{\sigma}{\Delta_{\phi}}\left(\left|\epsilon_{\phi_{i}}\right|+2 \sigma\right) & \text { for } \sigma \geq \Delta_{\phi}\end{cases}
$$

where,

$$
\Delta_{\phi}=\frac{\max \left(\phi_{i}\right)-\min \left(\phi_{i}\right)}{n_{g}-1}
$$

$U_{\text {disc }}$ is the discretization uncertainty of the $\phi_{i} . \sigma$ is the standard deviation of the least square estimation of the discretization error, which is multiplied by two to get a $95 \%$ confidence interval, due to the regression under the assumption of a normal distribution of the scatter in the simulation results. Finally, $n_{g}$ is the number of simulations used to determine the discretization error.

The discretization error is only determined over a single revolution of the propeller. The simulation should, therefore, have obtained periodic convergence before the simulation results are used. Periodic convergence means that the results for two propeller revolutions following each other should not change significantly. To determine if periodic convergence has been obtained for each simulation, the method proposed in [23] is used. In this method, a Fuzzy convergence set between 0 and 1 is defined, which should be larger than 0.95 for the simulation to have obtained periodic convergence. This Fuzzy convergence set considers the mean value, the discrete Fourier transform, the cross-correlation and power spectral density for each signal of the hydrodynamic loads. Hence, six signals are considered for each propeller blade per revolution to determine if the periodic convergence has been obtained. The results of the URANS CFD simulation of the CVP propeller are shown in Section 3 for the considered application. 


\section{Appendix B. Case Parameters for the CVP Model}

Table A1. Parameters used to model the loads acting on the CVP propeller blades in the considered case.

\begin{tabular}{lll}
\hline \multicolumn{2}{c}{ Propeller } \\
\hline Propeller diameter & $D_{p}$ & $5.4[\mathrm{~m}]$ \\
Number of blades & $Z_{b}$ & 4 \\
Power & & $5839[\mathrm{~kW}]$ \\
Hub diameter & & $1.46[\mathrm{~m}]$ \\
Blade area ratio & $n_{p}$ & 0.64 \\
Propeller speed & $h_{s}$ & $121[\mathrm{rpm}] \approx 2.02[\mathrm{rps}]$ \\
Shaft immersion & $\dot{\theta}_{b}$ & $5.07[\mathrm{~m}]$ \\
Blade speed & & $\approx 12.67[\mathrm{rad} / \mathrm{s}]$
\end{tabular}

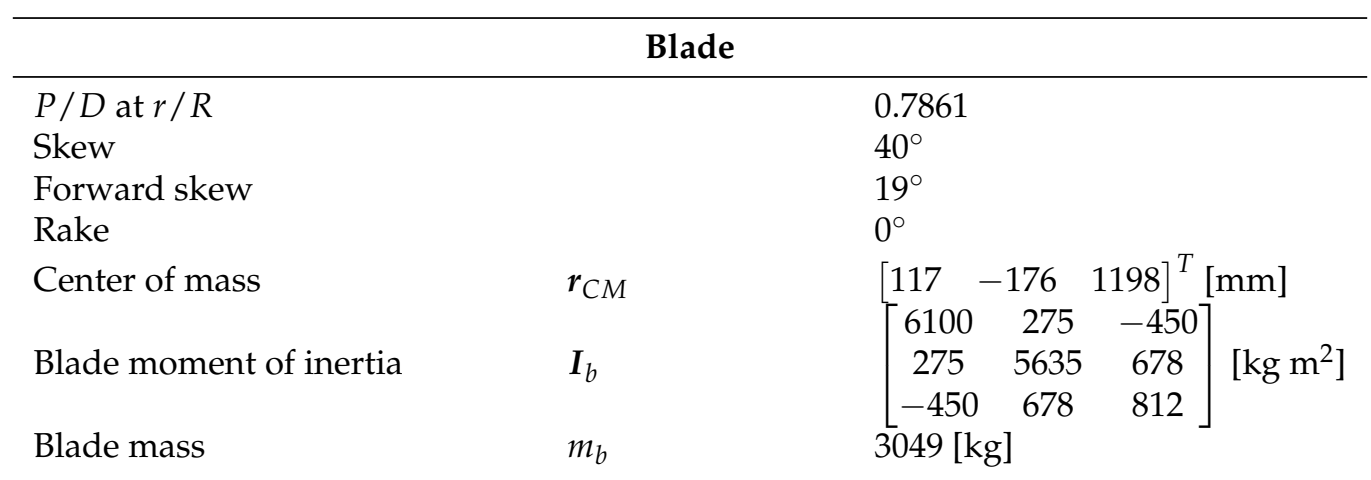

\begin{tabular}{lcl}
\hline & Operation Condition \\
\hline Ship speed & $V_{s}$ & $16.55[\mathrm{knots}]$ \\
& & $\approx 8.51[\mathrm{~m} / \mathrm{s}]$ \\
Water density & $\rho$ & $1025.5\left[\mathrm{~kg} / \mathrm{m}^{3}\right]$ \\
Kinematic viscosity of water & $v$ & $1.19110^{-6}\left[\mathrm{~m}^{2} / \mathrm{s}\right]$ \\
Nominal wake coefficient & $w_{\text {nom }}$ & 0.278 \\
Effective wake coefficient & $w_{\text {eff }}$ & 0.266 \\
& \multicolumn{2}{c}{ Misc } \\
\hline & \multicolumn{2}{c}{$412.5[\mathrm{~mm}]$} \\
\hline Inner blade bearing radius & $r_{b b}$ & $412.5[\mathrm{~mm}]$ \\
Outer blade bearing radius & $R_{b b}$ & $115[\mathrm{~mm}]$ \\
Height of blade bearing & $h_{b b}$ & {$[0 \quad 0 \quad 527.5]^{T}[\mathrm{~mm}]$} \\
Bearing translation & $r_{t}$ & 0.15 \\
Coulomb friction coefficients & $\mu_{c}$ & \\
\hline
\end{tabular}

\section{References}

1. Freiberg, U.S.; Andersen, T.O.; Nielsen, J.R. Review of Cyclic Varying Pitch Propeller for Commercial Vessels. In Sixth International Symposium on Marine Propulsors; CNR-INM, SINTEF Ocean and NTNU: Rome, Italy, 2019.

2. Takinaci, A.C.; Atlar, M. Performance assesment of a concept propulsor: The thrust-balanced propeller. Ocean. Eng. 2002, 29, 129-149. [CrossRef]

3. Simonsson, P. The pinnate propeller-A cavitation and noise-free propeller. In Proceedings of the Propeller ' 81 Symposium, Virginia Beach, VA, USA, 26-27 May 1981.

4. Simonsson, P. The Pinnate Propeller. Ph.D. Thesis, KTH Royal Institute of Technology, Stockholm, Sweden, 1983.

5. Simonsson, P. Report on Full-Scale Tests With Pinnate Propellers in a Swedish Navy Patrol Boat. In Proceedings of the Propeller '84 Symposium, Virginia Beach, VA, USA, 15-16 May 1984.

6. Jessup, S.D. Reduction of Propeller Vibration and Cavitation by Cyclic Variation of Blade Pitch; Massachusetts Institute of Technology: Cambridge, MA, USA, 1976. 
7. Gabriel, R.; Atlar, M. Calculation of the performance of a ship propeller with cyclic blade pitch control. Int. Shipbuild. Prog. 1998, 45, 201-223.

8. Turbo, M.D. Variable Pitch Setting During one Rotation. In Internal Report at MAN Diesel E Turbo Frederikshavn; MAN Diesel \& Turbo: Frederikshavn, Denmark, 2011; Revised.

9. UNCTAD. Review of Maritime Transport 2016; United Nations Conference on Trade and Development: Geneva, Switzerland, 2016.

10. Pronk, C. Blade Spindle Torque and Off-Design Behaviour of Controllable Pitch Propellers; TU Delft, Delft University of Technology: Delft, The Netherlands, 1980.

11. Thomsen, J.S.; Freiberg, U. Design of Shadow Pitpitch System for Controllable Pitch Propellers. Master's Thesis, Aalborg University, Aalborg, Denmark, 2015.

12. Freiberg, U.S.; Andersen, T.O.; Nielsen, J.R. Hydrodynamic Modelling and Performance Evaluation of Cycling Varying Pitch Propeller in Non-Uniform Wake Field Using Open-Water RANS CFD Simulations. In Proceedings of the International Conference on Offshore Mechanics and Arctic Engineering, Madrid, Spain, 17-22 June 2018; Volume 7A: Ocean Engineering. [CrossRef]

13. Martelli, M.; Figari, M.; Altosole, M.; Vignolo, S. Controllable pitch propeller actuating mechanism, modelling and simulation. J. Eng. Marit. Environ. 2013, 228, 29-43. [CrossRef]

14. Tarbiat, S.; Ghassemi, H.; Fadavie, M. Numerical Prediction of Hydromechanical Behaviour of Controllable Pitch Propeller. Int. J. Rotating Mach. 2014, 2014. Available online: https://www.hindawi.com/journals/ijrm/2014/180725/ (accessed on 11 October 2021). [CrossRef]

15. Godjevac, M.; van Beek, T.; Grimmelius, H.T.; Tinga, T.; Stapersma, D. Prediction of fretting motion in a controllable pitch propeller during service. J. Eng. Marit. Environ. 2008, 223, 541-560. [CrossRef]

16. Godjevac, M. Wear and Friction in a Controllable Pitch Propeller. Ph.D. Thesis, Delft University of Technology, Delft, The Netherland, 2010; ISBN: 978-90-8891-136-1.

17. Bakker, J.C.; Grimmelius, H.T.; Wesselink, A. The use of non linear model in the analysis of CPP actuator behaviour. In Proceedings of the World Maritime Technology Conference, London, UK, 6-10 March 2006.

18. Wesselink, A.F.; Stapersma, D.; van den Bosch, D.; Teerhuis, P.C. Non-linear aspects of propeller pitch control. In Proceedings of the World Maritime Technology Conference, London, UK, 6-10 March 2006.

19. ITTC Association. The Propulsion Committee: Final Report and Recommendations to the 24th ITTC. In Proceedings of the 24th ITTC (International Towing Tank Conference), Edinburg, Scotland, 1 October 2005.

20. Shin, K.W.; Regener, P.B.; Andersen, P. Methods for Cavitation Prediction on Tip-Modified Propellers in Ship Wake Fields. In Proceedings of the Fourth International Symposium on Marine Propulsors, Austin, TX, USA, 31 May-4 June 2015.

21. Wind, J. Principles of mechanisms used in controllable pitch propellers. Int. Shipbuild. Prog. 1971, 18, 80-94. [CrossRef]

22. Eca, L.; Hoekstra, M.; Vaz, G. Verification of Solutions in Unsteady Flows. In Proceedings of the ASME 2015 V\&V Symposium, Las Vegas, NV, USA, 13-15 May 2015.

23. Clark, J.; Grover, E. Assessing convergence in predictions of periodic-unsteady flowfields. J. Turbomach. 2007, 129, 740-749. [CrossRef] 